\title{
La meta-evaluación en la agenda de la educación superior
}

\author{
Meta-Evaluation in the Higher Education Agenda \\ A meta-avaliação na agenda da educação superior
}

Susana Marcipar Katz

Facultad de Ciencias Económicas,

Universidad Nacional del Litoral, Argentina.

E-mail:susmarcikatz@gmail.com

\author{
María de las Mercedes Luciani \\ Facultad de Ciencias Económicas, \\ Universidad Nacional del Litoral, Argentina. \\ E-mail: mercedesluciani@gmail.com
}

Fecha de recepción: 29/03/2017 Fecha de aceptación: 16/06/2017
Palabras clave - meta-evaluación

- aprendizaje

- enseñanza

- educación superior

- ciencias económicas

\section{Resumen}

En base a la influencia que ejerce la evaluación de los aprendizajes sobre los procesos educativos es que se torna relevante la idea de evaluar a las evaluaciones de los aprendizajes para rescatar a la evaluación como herramienta de cambio en la educación (Ravela, 2009). El artículo pretende difundir el diseño metodológico para aplicar una metaevaluación a dos asignaturas, Inglés Técnico y Matemática Básica, correspondientes a la carrera de Contador Público Nacional de la Universidad Nacional del Litoral. El objetivo investigativo es evaluar a las evaluaciones de los aprendizajes, meta-evaluación, en las asignaturas mencionadasa fin de lograr dos cuestiones. Por una parte, identificar los refuerzos o cambios necesarios que mejoren la calidad de los procesos de evaluación de manera tal que se constituyan en evaluación como aprendizaje, evaluación para el aprendizaje y evaluación de los aprendizajes. Por otra parte, determinar las condiciones epistemológicas, didácticas, culturales y tecnológicas adecuadas para promover el mejoramiento de la enseñanza y el aprendizaje en dichas asignaturas. Todo el diseño metodológico responde a las características de una evaluación iluminativa (Parlett y Hamilton, 1972), que se asume como una investigación orientada al descubrimiento y orientada a la aplicación (Arnal et ál., 1992). El diseño presentado de meta-evaluación puede convertirse en una estrategia institucional para la toma de decisiones académicas con miras a la construcción permanente de una cultura de auto-reflexión de la calidad de los aprendizajes. 
Keywords

- meta-evaluation

- learning

- teaching

- higher education

- economic science

\begin{abstract}
Based on the impact of learning assessment on educational processes, the idea of assessing learning evaluations to turn evaluation into a tool for change in education becomes relevant (Ravela,2009). This article intends to communicate the methodological design to apply a meta-evaluation on two subjects, English for Special Purposes and Basic Mathematics, which belong to the Accounting studies at Universidad Nacional del Litoral. The investigative objective is to evaluate the learning evaluations, i.e. metaevaluation, in the above mentioned subjects to reach two goals. On the one hand, identify the reinforcement or changes necessary to improve the quality of the evaluation processes so that they become evaluation as learning, evaluation for learning and evaluation of learning. On the other hand, determine the epistemological, didactic, cultural and technological conditions adequate to promote the improvement of teaching and learning in those subjects. The complete methodological design has the characteristics of an illuminative evaluation (Parlett and Hamilton, 1972), which assumes a research line oriented to discovery and application (Arnal et ál., 1992). The meta-evaluation design presented here can become an institutional strategy for academic decision-making that envisages the continuous construction of a culture of self-reflection on learning quality.
\end{abstract}

\section{Resumo}

Considerando a influência que exerce a avaliação das aprendizagens sobre os processos educativos é que se torna relevante a ideia de avaliar as avaliações das aprendizagens para resgatar a avaliação como ferramenta de mudança na educação (Ravela,2009). 0 artigo tenta difundir 0 desenho metodológico para aplicar uma meta-avaliação a duas disciplinas, Inglês Técnico e Matemática Básica, pertencentes ao curso de Contabilista Público Nacional da Universidade Nacional do Litoral. 0 objetivo da pesquisa é avaliar as avaliações das aprendizagens, meta-avaliação, nas disciplinas já citadas para conseguir duas coisas. Por uma parte, identificar os reforços ou mudanças necessários que melhorem a qualidade dos processos de avaliação de modo que se constituam em avaliação como aprendizagem, avaliação para a aprendizagem e avaliação das aprendizagens. Aliás, determinar as condições epistemológicas, didáticas, culturais e tecnológicas adequadas para promover a melhora do ensino e a aprendizagem em ditas disciplinas. 0 desenho metodológico todo responde às características de uma avaliação iluminativa (Parlett e Hamilton, 1972), que se toma como uma pesquisa orientada ao descobrimento e orientada à aplicação(Arnal, et al., 1992). 0 desenho de meta-avaliação apresentado pode se transformar em uma estratégia institucional para a toma de decisões acadêmicas a fim de promover a construção permanente de uma cultura de autorreflexão da qualidade das aprendizagens.
Palavras-chave

- meta-avaliação - aprendizagem - ensino

- educação superior - ciências econômicas. 


\section{A modo de introducción}

Nadie discute que el aprendizaje es el núcleo de la acción educativa. Sin embargo, son numerosos los autores que, investigando las actuales prácticas educativas, señalan a la evaluación de los aprendizajes como condicionante de las dinámicas de los procesos de enseñanza y de aprendizaje. Así, los estudiantes estudian tratando de recordar las cuestiones que «entran» en los temarios de examen. Por su parte, los docentes en sus clases ponen énfasis en los tópicos que serán objeto de evaluación. Entonces, la evaluación aparece convertida en eje pedagógico y bien podría decirse que en la Universidad, la hora de la verdad no es la del aprendizaje sino la de la evaluación.

Desde una perspectiva general los trabajos presentados por Brown y Glasner (2003) así como los de Hernández Pina (2002), Veres (2002), Cabrera, Bethencourt, Alvarez Pérez y González Afonso (2006) o Cabrera, Bethencourt, González Alfonso y Alvarez Pérez (2006) son claros exponentes del interés por la investigación sobre la educación superior en general. De forma específica, también son cada vez más frecuentes las publicaciones científicas focalizadas sobre la evaluación del aprendizaje en la educación superior, llegando autores como Trillo y Porto (2002:293) a «preconizar la evaluación de estudiantes como una línea relevante en el estudio de la calidad universitaria».

Estos autores presentan una excelente revisión sobre las investigaciones realizadas hasta ese momento en torno a la Evaluación del Aprendizaje en la Universidad. Desde una perspectiva más actualizada podemos citar las interesantes aportaciones de Boud y Falchikov (2007) o Falchikov (2005), además de los últimos números de las revistas especializadas en el ámbito de la evaluación y de la educación superior.

Dichos trabajos dan cuenta de que en los entornos educativos donde la evaluación instalada como práctica común es la tradicional —la que asocia evaluación a examen y se reduce a uno 0 varios exámenes para decidir sobre la promoción
0 acreditación de una asignatura-, los estudiantes no conocen los detalles de su actuación ni tienen consciencia de los saberes construidos, sólo les interesa el resultado final reflejado en notas 0 calificaciones. A su vez, los docentes no realizan ningún proceso sistematizado de aprendizaje personal de los resultados obtenidos en estos exámenes y la única actuación posterior que realizan es archivarlos 0 guardarlos por un determinado tiempo según las normativas vigentes. Sin duda, este es el escenario construido por concepciones dominantes en el nivel superior en Argentina y en otros países latinoamericanos sobre la evaluación de los aprendizajes, que están emparentadas conceptualmente a la evaluación sumativa en los términos de Michael Scriven (1967). Sin embargo, existen en la actualidad sistemas de evaluación en el nivel superior que dan cuenta de concepciones y prácticas superadoras.

\section{Sobre meta-evaluaciones educativas}

Así como meta-lenguaje es el lenguaje que se utiliza para hablar del lenguaje, la meta-evaluación remite a la evaluación aplicada a una evaluación realizada sobre un objeto, ente 0 sobre alguna actividad humana.

Entre las obras escritas por los exponentes de las teorías clásicas de la evaluación son escasas las referidas a meta-evaluación, pero hay coincidencias en atribuirle a Michael Scriven la paternidad del término. Así, Patricia Rogers (1997) cita un artículo de Scriven, «An Introduction to meta-evaluation», publicado en Educational Product Report en 1969. Una presentación formal del concepto metaevaluación se encuentra en el Evaluation Thesaurus de Scriven (1991). En este escrito, el autor discute la importancia y las implicaciones de realizar metaevaluaciones tanto formativas como sumativas, y presenta una idea central de «la meta-evaluación como imperativo profesional» de la disciplina, 
ya que considera que la responsabilidad de los resultados de una evaluación de cualquier actividad humana debe recaer, en primer término, en el diseño y aplicación de la propia evaluación. En dicho texto, Scriven considera a la meta-evaluación como una fase terminal del trabajo de evaluación. Para él, la evaluación de la evaluación consiste en la aplicación de listas de comprobación para construir un juicio sobre el resultado de la evaluación original. A través de dichas listas se obtiene una descripción meticulosa del proceso de evaluación y de los diversos elementos que han intervenido en la evaluación original. La más conocida es la denominada KEC (por Key Evaluation Checklist). En referencia a KEC, Luis Fernando Diaz (2001:172) afirma «esta lista es todavía preliminar y no cubre todo el temario que se esperaría encontrar en el campo de la meta-evaluación en la actualidad [...] una lista similar había sido preparada en la Western Michigan University en 1975, por Daniel Stufflebeam (MetaEvaluación)».

Con posterioridad han surgidos escritos sobre meta-evaluación, tal como la sección que dedica Ernest House en su libro Evaluación, Ética y Poder (Madrid, 1994). Otra obra que pone su atención en la meta-evaluación es la escrita por Schwandt y Halpem, Linking Auditing and Metaevaluation (1988). Ambos textos coinciden en otorgarle suma importancia a las etapas de preparación y elaboración previa a la aplicación de la meta-evaluación de modo que ésta arroje la información, aprendizaje y entendimientos que se desee hallar respecto de la evaluación original.

En 1999, Miguel Ángel Santos Guerra publica «Meta evaluación: rigor, mejora, ética y aprendizaje» donde elabora la noción de meta-evaluación comparándola sistemáticamente al concepto de evaluación enfatizando las distinciones epistemológicas.

En el año 2007, se presenta en la Universidad Autónoma de Barcelona una Tesis Doctoral de Martha Cruz Ávila titulada «Una propuesta para la evaluación del Profesorado Universitario». Si bien, en dicho título no se utiliza el concepto de metaevaluación, los dos primeros capítulos corresponden a un análisis crítico (a modo de evaluación) de los sistemas de evaluación de Profesores Universitarios existentes en España y en México. Es decir, la nueva propuesta que realiza Martha Cruz Ávila se sustenta en la evaluación que ella realiza respecto de las existentes evaluaciones de los profesores. En ese sentido, los dos primeros capítulos de su Tesis Doctoral pueden considerarse como meta-evaluación de los sistemas de evaluación de los Profesores Universitarios de España y México. La mención a esta tesis tiene un carácter ejemplificador en el contexto del presente artículo ya que muestra una nueva propuesta como dependiente, asociada y vinculada al diagnóstico que se realiza de una situación original (justamente la que se desea cambiar).

Por otra parte, en el año 2003 el Instituto Internacional de Planeamiento de la Educación IIPE UNESCO - Sede Regional Buenos Aires, edita el documento «Evaluar las Evaluaciones: Una mirada política acerca de las evaluaciones de la calidad educativa». En este documento se realizan metaevaluaciones desde una perspectiva política-pedagógica referida a las evaluaciones estandarizadas de la enseñanza primaria y secundaria. Es decir, realizados los procesos de evaluación, la metaevaluación incorpora como objeto a evaluar a dichos procesos de evaluación.

La evaluación como campo disciplinar, asociada o no a la acreditación, ha tenido un desarrollo exponencial en la Educación Superior en los últimos veinte años. En países como Francia, Suecia, Noruega, y más recientemente en Uruguay y Argentina, se han puesto en marcha mecanismos institucionales, centros u organismos de evaluación de los sistemas educativos. Se han desarrollado planes sistemáticos de evaluación en Inglaterra, Holanda, Francia, Argentina, México y República Dominicana. Se han elaborado indicadores de calidad educativa en Estados Unidos, Francia, Dinamarca y Suiza. Organismos Internacionales vinculados al desarrollo de políticas, como la Organización 
para la Cooperación y el Desarrollo Económico (OCDE), UNESCO, Unión Europea, la Organización de Estados Iberoamericanos (OEI), han puesto en marcha programas vinculados al desarrollo de políticas de evaluación educativa. Pero, en la actualidad todos esos programas y sistemas de evaluación están siendo objeto de evaluación. Así, en los últimos diez años la meta-evaluación o evaluación de las evaluaciones de calidad educativa pone su macro mirada para responder a interrogantes que van desde las cuestiones técnicas-pedagógicas a cuestiones socioeconómicas, políticas e ideológicas tales como: ilos instrumentos están midiendo correctamente los resultados?, ilos mecanismos de evaluación de la calidad educativa han provocado mayor responsabilidad por los resultados 0 han servido, por el contrario, para segmentar aún más la demanda educativa?, ilos mecanismos de evaluación que se aplican, son evaluaciones 0 son meras mediciones?, ¿evaluamos para mejorar la eficiencia de la inversión educativa o para orientar la demanda?, ievaluamos para compensar las diferencias sociales o para comprobar los niveles de segmentación social? Estos interrogantes, entre otros, indican un hecho que es innegable respecto de los sistemas de evaluación de la calidad educativa y es que ellos responden a diferentes modelos, que dan centralidad a la idea de equidad, otros que ponderan el concepto de competitividad y están aquellos que se centran en la eficiencia. Entonces, ya no se trata de considerar simplemente los resultados de una evaluación de calidad educativa sino que necesitamos evaluar a la evaluación que generó dichos resultados para comprender el sentido de esos resultados. Es decir, se torna necesario e importante la meta-evaluación del sistema de evaluación, ya que la evaluación educativa (sea sobre calidad o sobre los aprendizajes) se ocupa de los resultados del proyecto educativo. En cambio, a la meta-evaluación le interesan los resultados solo en cuanto síntoma del proyecto evaluado.

En este sentido, un ejemplo clarificador es el documento publicado en julio de 2015 por la
Universidad de Granada, España, titulado Criterios de evaluación de los proyectos de investigación: retos y excelencia a través de los informes de los evaluadores (Gómez, 2015). Se trata de una metaevaluación aplicada a los informes de evaluación recibidos por los investigadores de la Universidad de Granada. En esencia, se trata de conocer cómo se trasladan los criterios de evaluación publicados en las convocatorias a los informes que reciben los investigadores y cuáles son los apartados que reciben una mayor atención a fin de determinar los factores de éxito.

En resumen, desde una perspectiva epistemológica se caracteriza a la meta-evaluación, según diferentes autores, de los siguientes modos:

«La meta-evaluación trata de avivar la reflexión para generar comprensión rica y relevante sobre los programas evaluados» (Perkins, 1985:45).

«Meta-evaluar es un proceso social de aprendizaje quizás no tan complejo ni tan rico como la propia evaluación, pero no menos importante. Por ello [... ] se necesita algo más que un conjunto bien estructurado o una lista de criterios, se necesita que el meta-evaluador, al menos, plantee interrogaciones, sugerencias y perspectivas que alimenten la comprensión misma de los evaluadores y de la audiencia» (Santos Guerra, 1999:267).

«La meta evaluación consiste en la comprensión e interpretación de la evaluación. Busca la comprensión rica, ética y política de la evaluación. Por ello, la meta-evaluación implica y presupone retroalimentación y aprendizaje» (Diaz, 2001:177).

\section{Primer acercamiento al aprendizaje que surge de las evaluaciones}

En base a la influencia que ejerce la evaluación de los aprendizajes sobre los procesos educativos es que se torna relevante la idea de evaluar a las evaluaciones de los aprendizajes para rescatar a la «evaluación como herramienta de cambio en la 
educación» (Ravela, 2009), obteniendo de la metaevaluación las reflexiones adecuadas y pertinentes que iluminen el cambio que sea necesario realizar en las asignaturas.

Tal como se expuso anteriormente, coexisten tendencias diferentes sobre los sistemas de evaluación de los aprendizajes en el nivel superior. Por una parte los ya caracterizados sistemas tradicionales y por otra parte varios sistemas innovadores. Tal es lo observado en el informe publicado en el 2012 por el Board of Studies, Teaching \& Educational Standards, Australia, donde se infiere la necesidad de asumir en los ámbitos de educación superior una triple concepción evaluativa que denominan (assessment as learning, assessment for learning and assessment of learning) y que se traduce: evaluación como aprendizaje, evaluación para el aprendizaje y evaluación de los aprendizajes. Así, esa triple concepción se caracteriza de tal forma que ofrece a docentes y estudiantes la posibilidad de comprender diferentes cuestiones del proceso educativo y requiere actuar en consecuencia. Es decir, en dicho documento se caracteriza a cada una de estas evaluaciones y que, adaptadas al presente artículo, se traducen del siguiente modo:

Evaluación para el aprendizaje: proporciona a los profesores información sobre el conocimiento y competencias de los estudiantes para ser utilizada como input en la planificación de su desempeño docente, considerando que en dicho desempeño se incluyen las acciones que permitan a los estudiantes recibir el feedback adecuado para el mejoramiento de sus aprendizajes. Es decir, con este enfoque se obtiene información cuyos destinatarios son tanto los docentes como los estudiantes ya que posibilita la oportunidad de aprendizaje para ambos. Esto es evaluación para el aprendizaje de docentes y estudiantes.

Evaluación como aprendizaje: proporciona a los estudiantes las herramientas para la autorreflexión de los avances y déficit en los aprendizajes. Utilizan el feedback de los docentes para identificar su déficit y/0 errores de manera tal que monitorean y hacen conscientes sus propios procesos de apren- dizajes. Es un enfoque centrado en otorgar información a los estudiantes; es decir, la evaluación como el aprendizaje de los estudiantes.

Evaluación de los aprendizajes: proporciona a los docentes algunos elementos para estimar 0 apreciar los logros de los aprendizajes de los estudiantes de acuerdo con los objetivos y estándares establecidos, en nuestro caso, referidos a las carreras de grado de la Facultad de Ciencias Económicas de la Universidad Nacional del Litoral. Este enfoque tiene como usuario principal al docente quien diseña y desarrolla la evaluación de los aprendizajes para tomar decisiones de acreditación en las respectivas asignaturas.

Así, es posible plantear una sucesión de supuestos (S1...Sn) que conforman un determinado razonamiento deductivo, a modo de aproximación de hipótesis, que se enuncian del siguiente modo:

S1: La evaluación entendida de manera tradicional es un condicionante que restringe el desarrollo de evaluaciones «para», «como» $y$ «del» aprendizaje.

S2: Las evaluaciones "para», "como» y «del» aprendizaje son herramientas de cambio en el proceso educativo ya que cada una de ellas brinda información respecto de los procesos de enseñanza, de los aprendizajes y de las propias evaluaciones.

S3: Transformar la evaluación tradicional en un proceso de evaluación «para», «como» y «del» aprendizaje requiere de cambios que van desde las visiones y concepciones epistemológicas que tienen los docentes respecto de las disciplinas, pasando por lo que significa su aprendizaje y su enseñanza, hasta incluso aquellos cambios que impliquen adecuaciones tecnológicas y/0 logísticas apropiadas a cada asignatura para tal transformación.

S4: La evaluación de los sistemas de evaluación de los aprendizajes, meta-evaluación de los aprendizajes, en una determinada asignatura, permite obtener información tanto sobre el propio sistema de evaluación como así también sobre las fortalezas y las debilidades de los sistemas de enseñanza y de aprendizaje de dicha asignatura. 
S5: La meta-evaluación de los aprendizajes en una asignatura puede permitir la determinación de las condiciones epistemológicas, culturales, humanas, tecnológicas y logísticas necesarias para cambiar tanto el propio proceso de evaluación en evaluaciones «para» «como»y «del» aprendizaje, como para introducir los cambios necesarios a nivel de procesos de enseñanza y de aprendizaje.

Los anteriores supuestos (S1 a S5) conducen a establecer al menos la siguiente conclusión:

Si se aspira a generar cambios en los distintos procesos educativos de manera tal que se mejore su calidad es necesario determinar cuáles son los componentes, variables y constantes que deben ser modificados o fortalecidos. Entonces, la metaevaluación de los aprendizajes debería permitir la determinación de las condiciones epistemológicas, didácticas, culturales, humanas, tecnológicas y logísticas necesarias para cambiar tanto el propio proceso de evaluación como los de enseñanza y de aprendizaje.

\section{De la teoría a la práctica}

Las consideraciones anteriores se han constituido en el basamento teórico para diseñar y poner en marcha un proceso de meta-evaluación de los aprendizajes en dos asignaturas del Plan de Estudio de la carrera Contador Público Nacional de la FCE de la UNL, como lo son Inglés Técnico (3er año) y Matemática Básica (1er año). Dicho proceso se origina a mediados del año 2016 desde el Observatorio Académico de esa Facultad y para los primeros meses del 2017 se logra tanto consolidar un equipo de trabajo como también el diseño del proyecto para aplicar la meta-evaluación en las asignaturas mencionadas.

A continuación se describen las condiciones y características de ambos logros: equipo de trabajo y diseño del proyecto.

\section{Sobre el equipo de trabajo}

Todos los integrantes del equipo responsable de llevar adelante la meta-evaluación pertenecen a la planta permanente de la FCE de la UNL y trabajan en las áreas de Inglés o de Matemática e Informática. Como grupo, el estado de desarrollo en el tema específico de meta-evaluación es incipiente. Sin embargo, los integrantes cuentan individualmente con antecedentes relevantes en Investigación Educativa que garantizan el logro de los objetivos planteados.

Los criterios generales utilizados para conformar el equipo se basan en: a) pertinencia disciplinar; b) pertinencia metodológica; c) coherencia epistemológica.

a) Pertinencia disciplinar: Dado que esta investigación considera como objeto empírico a las asignaturas Inglés y Matemática, se han incorporado al equipo las profesoras titulares de ambas asignaturas y el titular de Informática quien articula ambas disciplinas. Además, están incluidas dos profesoras Adjuntas de Matemática y auxiliares docentes de ambas asignaturas.

b) Pertinencia metodológica: Dado que el diseño metodológico de la meta-evaluación posee tanto un carácter cualitativo como cuantitativo, los integrantes del equipo garantizan experiencia en dos sentidos. Uno de ellos es en el uso de medios tecnológicos capaces de realizar adaptaciones de software para permitir un eficiente y sustentable procesamiento de datos. El otro sentido es que los integrantes cuentan con experiencia 0 antecedentes en metodologías de investigación en educación.

c) Coherencia epistemológica: Más allá de asumir un posicionamiento pluralista y abierto respecto de diferentes paradigmas de investigación educativa -interpretativo, sociocrítico o constructivistaes necesario contar con un equipo de coordinación que posea coherencia respecto de creencias y valores como visión compartida de la educación en general y de la educación disciplinar. Dicha visión compartida está garantizada en la mayoría de los integrantes del equipo. 


\section{Sobre el diseño del proyecto}

Se ha definido como objetivo general evaluar a las evaluaciones de los aprendizajes, meta-evaluación, en las asignaturas de Inglés y Matemática de la FCE de la UNL a fin de identificar dos cuestiones, por una parte los refuerzos o cambios necesarios que mejoren la calidad de los procesos de evaluación, y por otra parte determinar las condiciones epistemológicas, didácticas, culturales y tecnológicas adecuadas para promover el mejoramiento de la enseñanza y el aprendizaje en dichas asignaturas. Y para su logro se han determinado los siguientes objetivos específicos:

- Diseñar y aplicar un sistema de meta-evaluación de los aprendizajes en Inglés y Matemática bajo condiciones tecnológicas y logísticas que le otorguen sustentabilidad.

- Interpretar los datos e información obtenida de la meta-evaluación para identificar las fortalezas y las debilidades de los sistemas evaluados, consensuando al interior de las asignaturas los cambios factibles de realizar que tiendan a la mejora de dichos sistemas.

- Identificar, desde la meta-evaluación, las condiciones epistemológicas, didácticas, culturales y tecnológicas al interior de las asignaturas Inglés y Matemática que más puedan estar incidiendo en los procesos educativos respectivos para mejorar el aprendizaje y la enseñanza de ambas asignaturas.

- Crear una cultura de autorreflexión en los docentes y en los estudiantes respecto de los procesos de evaluación, de aprendizaje y de enseñanza teniendo en cuenta la meta-evaluación aplicada.

Para el logro de dichos objetivos se requiere de un adecuado diseño metodológico en congruencia con el marco teórico, los supuestos o hipótesis y el problema e interrogantes que se desean responder. De tal manera, resulta importante rescatar las perspectivas teóricas a las que se adhiere junto a los supuestos establecidos y los objetivos, todo ello ya enunciado con anterioridad pero ahora desde su abordaje metodológico.

\section{El problema y las preguntas metodológicas}

La importancia que la Universidad y la comunidad universitaria le asignan a las evaluaciones de los aprendizajes de los estudiantes no concuerda con la utilización que de sus resultados se hace para definir políticas educativas estructurales 0 para tomar decisiones al interior de una asignatura que procuren el mejoramiento de los procesos educativos. Esta escasa utilización de los resultados puede deberse, entre otras cuestiones, a que la evaluación de las evaluaciones (meta-evaluación) requiere de cuestiones que van desde la conformación de sólidos equipos técnicos hasta los acuerdos culturales que orienten lo que se pretende evaluar de las evaluaciones. En particular, al interior de una asignatura el conocimiento de los nudos críticos de su sistema de evaluación pone en alerta al propio sistema de enseñanza y de aprendizaje. Dicha situación de exposición o constatación del déficit del proceso educativo suele interpelar a los docentes de manera tal que sienten más una amenaza que una oportunidad de crecimiento en su profesión docente. Entonces, el problema no es únicamente establecer mecanismos capaces de revelar cuestiones susceptibles de mejora ya que una vez identificadas aparecen los condicionamientos culturales, humanos, epistemológicos y didácticos que pueden dificultar la concreción de la mejora. En este sentido, la meta-evaluación de los aprendizajes, concebida sistémicamente, si es relevante y bien utilizada puede convertirse en la principal estrategia para marcar el camino hacia la mejora permanente de la calidad de lo que se enseña, se aprende y se evalúa.

Analizando el objetivo general enunciado, se observa la pretensión de evaluar a los sistemas de evaluación de los aprendizajes en Inglés y Matemática para valorarlos, rescatarlos, ponderarlos como herramientas de cambio y transformación de los procesos educativos. Asimismo se reconoce que esa función transformadora solo es posible en determinadas condiciones. 
Al pretender identificar los mejores criterios, normas, funciones, fines y procedimientos de evaluación de las evaluaciones, o meta-evaluación, que permitan a los docentes y estudiantes acceder a una mayor comprensión de los procesos de aprendizaje y de enseñanza, se está sosteniendo que:

- «Si (...) se resuelve producir modificaciones en los sistemas de evaluación de los aprendizajes, éstos tienden a desarrollar una indudable influencia sobre las estrategias de enseñanza de los profesores y, particularmente, sobre las estrategias de aprendizaje de los alumnos, con un efecto denominado de validez retroactiva» (Camilloni, Celman, Litwin y Palou de Mat, 1998:133).

- Desde otro punto de vista, se está sosteniendo la necesidad de que, en los procesos de evaluación, debe existir una adecuada comunicación y devolución de los resultados y procesos obtenidos de las producciones que realizan los estudiantes. Esto significa, pasar de la evaluación de los aprendizajes a la evaluación para los aprendizajes (Perez Gomez, 2009:6), es decir, que se sustenta la idea de considerar a la evaluación como instrumento de aprendizaje en vez de un mero requisito de control y calificación.

- Como señala Shepard (2000), «si la evaluación tiene la finalidad de ayudar a los estudiantes a aprender, debe modificarse de dos formas fundamentales. Primero, el contenido y el carácter de las evaluaciones debe ser mejorado significativamente. Segundo, la información obtenida en la evaluación debe formar parte del proceso de aprendizaje».

Entonces, para el logro del objetivo general bajo los supuestos y sustentos teóricos aquí planteados, se definen los siguientes interrogantes desde un punto de vista metodológico:

(1) ¿Cómo identificar cuáles son los rasgos esenciales que debería tener la meta-evaluación de los aprendizajes en Inglés y en Matemática?
(2) ¿Cuáles son y cómo se determinan las condiciones tecnológicas sustentables que faciliten la aplicación y procesamiento de un sistema de metaevaluación de los aprendizajes en las asignaturas de Inglés y Matemática?

(3) ¿De qué manera es posible desarrollar condiciones culturales apropiadas para garantizar reflexiones en los docentes a cerca de cuestiones epistemológicas, didácticas y culturales referidas a las disciplinas, a su enseñanza, su aprendizaje y su evaluación?

Las tres preguntas anteriores orientan al diseño metodológico y son respondidas puntualmente una vez que se expliciten las características generales de la presente investigación.

\section{Actividades, Métodos y Técnicas}

Es importante señalar la diferencia que los autores realizan entre «evaluación» y «meta-evaluación». Aun cuando la temática que se aborda es la misma y ambas comparten las bases teóricas, se trata de procedimientos y estructuras de diseño diferentes y lo más importante es la caracterización del metaevaluador que se distingue profundamente del evaluador. El meta-evaluador es aquel que está «inmerso en un ambiente abierto de aprendizaje, formulando preguntas, aparentemente abiertas y poco estructuradas, aunque siguiendo en realidad una rica intuición, especie de corazonada informada y atenta a la experticia» (Santos Guerra, 1999:32).

Santos Guerra al referirse a la estructura y diseño de una meta-evaluación, establece secuencias temporales que denomina «momentos de la metaevaluación» y que se han respetado adaptándolos al caso de Inglés y Matemática, como se muestra en el siguiente esquema: 
Esquema 1. Itinerario metodológico

\begin{tabular}{ll}
\hline Constitución & 1. Génesis de la evaluación \\
& 2. Iniciativa gestada por los protagonistas \\
\hline Justificación & 3. Proceso de consensos \\
& 1. Estrategias, métodos y procedimientos \\
& 2. Tratamiento de los datos \\
& 3. Rigor referido a: \\
& - argumentación utilizada \\
& - selección y aplicación de los métodos \\
\hline Presentación & - triangulación \\
\hline 1. Contenido y forma de los informes \\
2. Adecuación a diversas audiencias \\
3. La estructura lógica, la coherencia interna, la extensión, la claridad expositi- \\
$\frac{\text { va, etcétera. }}{\text { Motivaciones, intenciones, actitudes, presiones, aprendizajes }}$ \\
\hline Carácter democrático y participativo: Aceptaciones y rechazos del sistema o de \\
\hline
\end{tabular}

Fuente: Elaboración propia.

El esquema anterior muestra a grandes rasgos el itinerario metodológico.

En sintonía con los tres interrogantes anteriormente expuestos, se identifican las actividades, los métodos y técnicas que permitirán hallar respuestas, a saber:

Respecto del interrogante (1): ¿Cómo identificar cuáles son los rasgos esenciales que debería tener la meta-evaluación de los aprendizajes en Inglés y en Matemática? corresponde ubicarlo en el primer momento del esquema, denominado «constitución»

La meta-evaluación debe constituirse de tal manera que sea capaz de detectar aquellos aspectos relevantes de los sistemas de evaluación que denoten síntomas de los procesos de enseñanza y de aprendizaje, en cada asignatura: Inglés y Matemática.

Para lograr consensos sobre los rasgos que se consideren esenciales, se desarrollan las siguientes actividades:

- Entrevistas en profundidad a todos los integrantes de las asignaturas de Inglés y Matemática, para identificar los diferentes propósitos, preocupaciones, dificultades, motivaciones que asocian al acto de «evaluar».

- Análisis e interpretación de las entrevistas agrupando los resultados obtenidos en categorías tales como: instrumentos de evaluación, elaboración de consignas, contenido de las evaluaciones, criterios de evaluación, escalas de evaluación, resultados de las evaluaciones, distribución de calificaciones, aspectos emocionales y demás categorías que puedan surgir de las entrevistas.

- Diseño y aplicación de encuestas semiestructuradas (con preguntas abiertas y cerradas) dirigidas a estudiantes para rescatar sus experiencias tanto formales como emocionales, obtenidas de las evaluaciones de cada asignatura, de modo que se observen las categorías anteriores u otras pero desde la perspectiva de los alumnos.

- Análisis e interpretación conjunta de las entrevistas a docentes y encuestas a estudiantes. Las respuestas obtenidas en ambos casos se analizarán utilizando métodos propios del Análisis del Discurso: 
identificación de categorías de análisis, posicionamientos idiosincráticos, interacciones intratextos, consistencias y diferencias entre los discursos de los participantes, etc. Estos análisis se potenciarán mediante técnicas basadas en corpus: diseño y digitalización de corpora especializado; análisis manual de los datos y análisis con software de corpus; obtención de concordancias para el análisis de las categorías indicadas en sus correspondientes entornos de us0; análisis de consistencia, entre otros.

- Identificación de los ejes de evaluación que deben estar presentes en el sistema de metaevaluación. Esta identificación será producto de los análisis e interpretaciones efectuadas en las actividades anteriores.

Respecto del interrogante (2) ¿Cuáles son y cómo se determinan las condiciones tecnológicas sustentables que faciliten la aplicación y procesamiento de un sistema de meta-evaluación de los aprendizajes en las asignaturas de Inglés y Matemática? Se lo ubica en el segundo momento del esquema 0 diagrama, denominado «justificación».

- La respuesta a dicho interrogante dependerá de los resultados obtenidos en la etapa anterior, pues la tecnología que se elija tendrá que contemplar el tratamiento de las decisiones asumidas respecto de los ejes de análisis y variables intrínsecas a ellos. Las acciones previstas son,

- Selección del software más apropiado según el tamaño de la base de datos que deba construirse.

- Conformación del equipo responsable para la carga de datos aun cuando se trate de diseños con lectores electrónicos de barra.

- Determinación y análisis de las triangulaciones que serán necesarias procesar de la matriz que se construya. - Análisis permanente de los resultados que se obtengan de la base de datos cualitativos y cuantitativos y de las triangulaciones.

El tercer interrogante (3) ide qué manera es posible desarrollar condiciones humanas apropiadas para garantizar reflexiones en los docentes a cerca de cuestiones epistemológicas, didácticas y culturales referidas a las disciplinas, a su enseñanza, su aprendizaje y su evaluación? Tendrá respuesta en los tres últimos momentos del diagrama: Presentación, Valoración y Revisión Circular. Para ello se realizarán las siguientes acciones:

- Encuentros grupales de los docentes de las respectivas asignaturas con técnicas propias de la psicosociología de las organizaciones: focus-group, torbellino de ideas, liderazgo del conocimiento, etc. En estos encuentros se diseñarán informes que reflejen las interpretaciones surgidas de los participantes respecto de los datos procesados en las etapas anteriores.

- Elaboración del contenido y forma que tendrá el feedback de los docentes a los estudiantes, para cada asignatura, según las interpretaciones de los docentes.

- Elaboración del contenido y forma que tendrá el feedback, al interior de cada asignatura, para superar 0 mejorar el proceso de evaluación y/0 de enseñanza y/o de aprendizaje.

- Elaboración de diferentes instrumentos de evaluación para y como aprendizaje, en cada una de las asignaturas

- Aplicación de las evaluaciones para y como aprendizaje

- Revisión circular del proceso de meta-evaluación para la reflexión y mejoras en cada asignatura.

En resumen, la secuencia de actividades a emprender para el logro del objetivo general es:

- Entrevistas en profundidad a todos los integrantes de las asignaturas de Inglés y Matemática,

- Análisis e interpretación de las entrevistas

- Diseño y aplicación de encuestas dirigidas a estudiantes

- Análisis e interpretación conjunta de las entrevistas a docentes y encuestas a estudiantes.

- Identificación de los ejes para la meta-evaluación

- Selección del software

- Conformación del equipo responsable para la carga de datos 
- Determinación y análisis de las triangulaciones

- Análisis permanente de los resultados parciales.

- Encuentros grupales de desarrollo personal con docentes de las respectivas asignaturas

- Elaboración del contenido y forma que tendrá el feedback de los docentes a los estudiantes, para cada asignatura.

- Elaboración del contenido y forma que tendrá el feedback, al interior de cada asignatura

- Elaboración y aplicación de evaluaciones para y como aprendizaje.

- Revisión circular del proceso de meta-evaluación.

\section{Reflexiones finales}

En Argentina se están realizando los acuerdos normativos y formales para concretar el proceso de «acreditación de las carreras de Contador Público Nacional» correspondientes a Universidades Nacionales tanto de gestión pública como de gestión privada. En estas circunstancias, se considera apropiada la difusión del concepto y sentido de la meta-evaluación con las múltiples aplicaciones que en la actualidad se realizan, y cada vez con mayor asiduidad, desde el mundo académico internacional.
Específicamente la meta-evaluación de los aprendizajes, concebida sistémicamente, si es relevante y bien utilizada puede convertirse en la principal estrategia para marcar el camino hacia la mejora permanente de la calidad de lo que se enseña, se aprende y se evalúa. Por ello, el caso comentado del diseño de meta-evaluación de los aprendizajes en Matemática e Inglés tiene carácter ilustrativo, no exhaustivo, de la complejidad en la que los procesos de evaluación están inmersos. Esa misma complejidad es la que justifica y pone en evidencia la necesidad de considerar a las evaluaciones de los aprendizajes como objeto de estudio y de reflexión sistematizada.

Asumir procesos de meta-evaluación es en esencia una estrategia para construir una cultura de auto reflexión en los diferentes estamentos que integran la comunidad universitaria. Su complejidad puede verse simplificada si se la concibe en una economía de escala ya que gran parte del proceso informático puede servir para varias asignaturas, adaptando los cambios intrínsecos a cada caso. Es entonces que, la meta-evaluación puede convertirse en una estrategia institucional para la toma de decisiones académicas con miras a la construcción permanente de una cultura de autorreflexión de la calidad de los aprendizajes.

\section{Referencias bibliográficas}

- Arnal, J., Del Rincón, D., y Latorre, A. (1992). Investigación Educativa: Fundamentos y metodologías. Barcelona, España: Labor.

- Board of Studies Teaching \& Educational Standards (2012). Informes institucionales. Australia. Recuperado el 15 de marzo de 2016 de http:// www.boardofstudies.nsw.edu.au)
- Boud, D., y Falchikov, N. (Eds.) (2007). Rethinking Assessment in Higher Education. Learning for the longer term. London: Routledge.

- Brown, S., y Glasner, A. (Ed.) (2003). Evaluar en la universidad. Problemas y nuevos enfoques. Madrid: Narcea.
- Cabrera, L., Bethencourt, J.T., Alvarez Pérez, P., y González Afonso, M. (2006). El problema del abandono de Ios estudios universitarios. RELIEVE, Revista Electrónica de Investigación y Evaluación Educativa, 12(2). Recuperado el 20 de abril de 2007 de: http://www.uv.es/RELIEVE/v12n2/ RELIEVEv12n2_1.htm 
- Cabrera, L., Bethencourt, J.T., González Alfonso, M., y Alvarez Pérez, P. (2006). Un estudio transversal retrospectivo sobre prolongación y abandono de estudios universitarios. RELIEVE, Revista Electrónica de Investigación y Evaluación Educativa, 12(1). Recuperado el 20 de abril de 2007 de http://www.uv.es/RELIEVE/ v12n1/RELIEVEv12n1_1.htm

- Camilloni, A.R.W., Celman, S., Litwin, E., y Palou de Mat, M. del C. (1998). La evaluación de los aprendizajes en el debate didáctico contemporáneo. Buenos Aires, Barcelona, México: Paidós.

- Cruz Ávila, M. (2007). Una propuesta para la evaluación del Profesorado Universitario (Tesis doctoral). Department de Pedagogia Sistemàtica i Social, Universitat Autònoma de Barcelona. Barcelona, España.

- Diaz, L.F. (2001). La metaevaluación y su método. Revista Ciencias Sociales, 2(3), 170-192. Costa Rica: Universidad de Costa Rica.

- Falchikov, N. (2005). Improving Assessment Through Student Involvement. Practical solutions for aiding learning in higher and further education. London: Routledge Falmer.

- Gómez, M. (2015, Julio). Criterios de evaluación de los proyectos de investigación: retos y excelencia a través de los informes de los evaluadores. Informe - 1013-10 Vicerrectorado de Investigación y Transferencia - Servicio de Gestión de la Investigación. Recuperado en Julio 2017 de http://investigacion.ugr.es/ugrinvestiga/pages/doc ugrcifras/criteriosdevaloracion21docx/ - Hernández Pina, F. (2002). Consistencia entre motivos y estrategias de aprendizaje en estudiantes universitarios. Revista de Investigación Educativa, 20(2), 487-510.
- House, E. (1994). Evaluación, ética y poder. Madrid: Morata.

- Instituto Internacional de Planeamiento de la Educación (IIPE) (2003). Evaluar las Evaluaciones: Una mirada política acerca de las evaluaciones de la calidad educativa. Buenos Aires: UNESCO. Recuperado en Julio 2017 de http://www. buenosaires.iipe.unesco.org/publicaciones/evaluar-las-evaluacionesuna-mirada-pol-tica-acerca-delas-evaluaciones-de-la-calidad

- Parlett, M., y Hamilton, D. (1972). Evaluation as illumination: A new approach to the study of innovatory programs. Edinburgh: University of Edinburgh, Centre for Research in the Educational Sciences.

- Perez Gomez, A.I. (2009). La evaluación como aprendizaje. España: Ediciones Akal.

- Perkins, D.N. (1985). Conocimiento como diseño. Bogotá: Pontificia Universidad Javeriana.

- Ravela, P. (2009). Evaluación como herramienta de cambio en la educación. En F. Martínez Rizo y E. Martín (Coord.) (2009), Avances y desafíos en la evaluación educativa, «Metas Educativas 2021». Organización de Estados Iberoamericanos (OEI) y Fundación Santillana.

- Rogers, P. (Comp.) (1997). Metaevaluation bibliography. Program for Public Sector Evaluation. Royal Melbourne Institute of Technology. Recuperado en Julio 2017 de http:// www.aral.com.au/resources/metaeval-bib.html

- Santos Guerra, M.A. (1999). Metaevaluación: rigor, mejora, ética y aprendizaje. Madrid: Síntesis.

- Schwandt, T.A., y Halpem, E.S. (1988). Linking Auditing and Metaevaluation: enhancing Quality in
Applied Research (Applied Social Research Methods). London, New Delhi: SAGE Publications.

- Shepard, L.A. (2000). The Role of Assessment in a Learning Culture. Educational Researcher, 29(7), 4-14. - Scriven, M. (1991). Evaluation Thesaurus (4ta. Edición). Newbury Park, London, New Delhi: Sage Publications.

- Scriven, M. (1967). The methodology of evaluation. En R. W. Stake et ál., Perspectives on curriculum evaluation (AERA, Monograph Series on Curriculum Evaluation, 1). Chicago: Rand MacNally.

- Trillo Alonso, F., y Porto Currás, M. (2002). La evaluación de los estudiantes en el marco de la evaluación de la calidad de las universidades. Revista de Educación, 328, 283-301.

- Veres, E. (2002). Los nuevos planes de estudio y su incidencia sobre la edad de finalización de los estudios superiores. El caso de la Universidad de Valencia. RELIEVE, Revista Electrónica de Investigación y Evaluación Educativa, 8(1), 30-48.

\section{Registro bibliográfico}

Marcipar Katz, S., y Luciani, M.M. (2017). La meta-evaluación en la agenda de la educación superior. Revista Ciencias Económicas, 14(01), 185-197. 